DESY 06-244

UT-07-03

February 2007

\title{
Gravitino Dark Matter in R-Parity Breaking Vacua
}

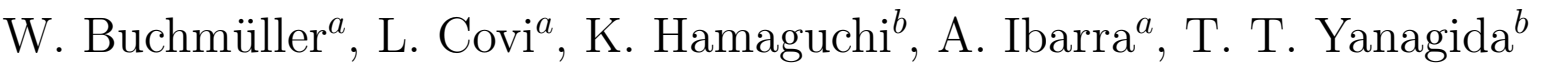 \\ a Deutsches Elektronen-Synchrotron DESY, Hamburg, Germany \\ b Department of Physics, University of Tokyo, Tokyo, Japan
}

\begin{abstract}
We show that in the case of small R-parity and lepton number breaking couplings, primordial nucleosynthesis, thermal leptogenesis and gravitino dark matter are naturally consistent for gravitino masses $m_{3 / 2} \gtrsim 5 \mathrm{GeV}$. We present a model where R-parity breaking is tied to B-L breaking, which predicts the needed small couplings. The metastable next-to-lightest superparticle has a decay length that is typically larger than a few centimeters, with characteristic signatures at the LHC. The photon flux produced by relic gravitino decays may be part of the apparent excess in the extragalactic diffuse gamma-ray flux obtained from the EGRET data for a gravitino mass $m_{3 / 2} \sim 10 \mathrm{GeV}$. In this case, a clear signal can be expected from GLAST in the near future.
\end{abstract}




\section{Introduction}

Most supersymmetric extensions of the standard model impose R-parity [1] as an exact symmetry of the supergravity Lagrangian. In this way, one forbids renormalizable baryon and lepton number violating interactions which might cause too rapid proton decay [2]. On theoretical grounds, however, theories with and without R-parity are on the same footing, and in low-energy effective theories obtained from string compactifications Rparity plays no preferred role.

One can also construct supersymmetric extensions of the standard model without R-parity [3], and the phenomenological constraints on these theories have been studied in great detail [4]. Without R-parity conservation, the lightest superparticle (LSP) is no longer stable and, in general, it does not contribute to dark matter.

Stringent constraints on the lepton number and R-parity violating interactions

$$
W_{\Delta L=1}=\lambda_{i k j} l_{i} e_{j}^{c} l_{k}+\lambda_{k j i}^{\prime} d_{i}^{c} q_{j} l_{k}
$$

are imposed by baryogenesis. Both operators contain lepton doublets. Together with sphaleron processes they therefore influence the baryon asymmetry at high temperature in the early universe. The requirement that an existing baryon asymmetry is not erased before the electroweak transition typically implies [5]

$$
\lambda, \lambda^{\prime}<10^{-7} \text {. }
$$

It is very remarkable that for such a small breaking of R-parity a gravitino LSP has a lifetime much longer than the age of the universe [6]. This is due to the double suppression by the inverse Planck mass and the R-parity breaking coupling, $\Gamma_{3 / 2} \propto \lambda^{2} m_{3 / 2}^{3} / M_{\mathrm{P}}{ }^{2}$. We find for the gravitino lifetime

$$
\tau_{3 / 2} \sim 10^{26} \mathrm{~s}\left(\frac{\lambda}{10^{-7}}\right)^{-2}\left(\frac{m_{3 / 2}}{10 \mathrm{GeV}}\right)^{-3},
$$

which is consistent with gravitino dark matter.

For a gravitino LSP, the properties of the next-to-lightest superparticle (NLSP) are strongly constrained by primordial nucleosynthesis (BBN). In the particularly interesting case of a charged NLSP, like a scalar $\tau$-lepton, its lifetime has to be relatively short, $\tau_{\mathrm{NLSP}} \lesssim 10^{3}-10^{4} \mathrm{~s}[7]$, which typically requires $m_{3 / 2}<1 \mathrm{GeV}$. Even for neutral particles, BBN excludes a neutralino NLSP for lifetimes longer than $10^{2} \mathrm{~s}$ due to the

\footnotetext{
${ }^{1}$ See also [8]. Here, we consider $m_{\mathrm{NLSP}}=\mathcal{O}(100 \mathrm{GeV})$. For a heavier charged NLSP, $m_{\mathrm{NLSP}}>$ $\mathcal{O}(1 \mathrm{TeV})$, the bound on the lifetime becomes even more stringent (cf. [9]). We do not consider a late time entropy production in this paper, which is an another possible way to avoid these BBN constraints [10].
} 
strong constraints from hadronic showers [9]. Only a sneutrino NLSP could be marginally acceptable also with longer lifetimes, and therefore larger gravitino mass, in the region where the hadronic branching ratio of the decay is below $10^{-3}$ [11].

On the other hand, standard thermal leptogenesis [12], an attractive model for baryogenesis, needs a large reheating temperature in the early universe, $T_{R} \gtrsim 10^{9} \mathrm{GeV}$ (cf. [13]14]). This reheating temperature implies $m_{3 / 2} \gtrsim 5 \mathrm{GeV}$ for a gluino mass of $m_{\tilde{g}}=500 \mathrm{GeV}$ in order to avoid overclosure of the universe due to thermal gravitino production $[1516]^{2}$. The lower bound on the gravitino mass scales as $m_{3 / 2}^{\min } \sim T_{R} m_{\tilde{g}}^{2}$.

All these cosmological problems are automatically solved without any fine tuning of parameters in the case of a small breaking of R-parity, as given in Eq. (2), with a gravitino LSP. The NLSP lifetime becomes sufficiently short for $\lambda, \lambda^{\prime}>10^{-14}$,

$$
\tau_{\mathrm{NLSP}} \simeq 10^{3} \mathrm{~S}\left(\frac{\lambda}{10^{-14}}\right)^{-2}\left(\frac{m_{\mathrm{NLSP}}}{100 \mathrm{GeV}}\right)^{-1} .
$$

Therefore, primordial nucleosynthesis, thermal leptogenesis and gravitino dark matter are naturally consistent for $10^{-14}<\lambda, \lambda^{\prime}<10^{-7}$ and $m_{3 / 2} \gtrsim 5 \mathrm{GeV}$. This is the main point of this paper.

The paper is organised as follows. In Sec. 2 we present a model where R-parity breaking is tied to B-L breaking, yielding the needed small R-parity breaking couplings. Sec. 3 deals with constraints from neutrino masses. Sec. 4 deals with implications for cosmology and collider physics. The results are discussed in Sec. 5.

\section{R-Parity Breaking and B-L Breaking}

\subsection{A Model of R-Parity Breaking}

We consider a supersymmetric extension of the standard model whose symmetry group $G$ includes $U(1)_{B-L}$ and R-invariance,

$$
G=S U(3) \times S U(2) \times U(1)_{Y} \times U(1)_{B-L} \times U(1)_{R}
$$

Three quark-lepton generations can be grouped into the $S U(5)$ representations $\mathbf{1 0}_{i}=$ $\left(q, u^{c}, e^{c}\right)_{i}, \overline{\mathbf{5}}_{i}=\left(d^{c}, l\right)_{i}$ and $\mathbf{1}=\nu_{i}^{c}$, which together form 16-plets of $S O(10)$. In addition, we have two Higgs doublets $H_{u}$ and $H_{d}$, two standard model singlets $N^{c}$ and $N$, and three

\footnotetext{
${ }^{2}$ We use the perturbative result for the gravitino production rate to leading order in the strong gauge coupling $g$. Since $g$ and also the thermal gluon mass are large, the perturbative expansion is problematic [15. The uncertainty due to higher orders in $g$ and nonperturbative effects is $\mathcal{O}(1)$. Possible effects due to thermal masses are also $\mathcal{O}(1)$ [17.
} 
$S O(10)$ singlets $X, \Phi$ and $Z$. The two Higgs doublets are contained in $\mathbf{5}$ - and $\overline{\mathbf{5}}$-plets of $S U(5)$, which we shall also denote as $H_{u}$ and $H_{d}$, respectively. $N^{c}$ and $N$ are contained in 16 and $\overline{\mathbf{1 6}}$ of $S O(10)$, which fixes their B-L charge to be +1 and -1 , respectively. $X$, $\Phi$ and $Z$ have B-L charge zero. This set of fields is familiar from $S O(10)$ orbifold GUTs (cf. [18]): matter fields form complete $S O(10)$ representations, whereas fields which break $S U(2) \times U(1)_{Y}$ and $U(1)_{B-L}$ appear as 'split' multiplets. For simplicity, we shall use in the following often $S U(5)$ notation also for the Higgs multiplets.

The matter sector of the superpotential has the usual form

$$
W_{M}=h_{i j}^{(u)} \mathbf{1 0}_{i} \mathbf{1 0} H_{j} H_{u}+h_{i j}^{(d)} \overline{\mathbf{5}}_{i} \mathbf{1 0}_{j} H_{d}+h_{i j}^{(\nu)} \overline{\mathbf{5}}_{i} \mathbf{1}_{j} H_{d}+\frac{1}{M_{\mathrm{P}}} h_{i j}^{(n)} \mathbf{1}_{i} \mathbf{1}_{j} N^{2},
$$

where $M_{\mathrm{P}}=2.4 \times 10^{18} \mathrm{GeV}$ is the Planck mass. The expectation values of the Higgs multiplets $H_{u}$ and $H_{d}$ generate Dirac masses of quarks and leptons, whereas the expectation value of the singlet Higgs field $N$ generates the Majorana mass matrix of the right-handed neutrinos $\mathbf{1}_{i}$. The superpotential responsible for B-L breaking is chosen as

$$
W_{B-L}=X\left(N N^{c}-\Phi^{2}\right)
$$

where unknown Yukawa couplings have been set equal to one. $\Phi$ plays the role of a spectator field, which will finally be replaced by its expectation value, $\langle\Phi\rangle=v_{B-L}$. Similarly, $Z$ is a spectator field 3 , which breaks supersymmetry and $U(1)_{R},\langle Z\rangle=F_{Z} \theta \theta$. The superpotential in Eqs. (6) and (17) is the most general one consistent with the R-charges listed in Table 1, up to higher order terms which we will discuss later. Note that the choice of a negative R-charge for $N^{c}$ forbids the dangerous superpotential terms

$$
\overline{\mathbf{5}}_{i} H_{d} N^{c}, \quad \overline{\mathbf{5}}_{i} \overline{\mathbf{5}}_{j} \mathbf{1 0} \mathbf{0}_{k} N^{c},
$$

which would yield too large bilinear mixings and too rapid proton decay, respectively.

The expectation value of $\Phi$ leads to the breaking of $B-L$,

$$
\langle N\rangle=\left\langle N^{c}\right\rangle=\langle\Phi\rangle=v_{B-L}
$$

where the first equality is a consequence of the $U(1)_{B-L}$ D-term. This generates a Majorana mass matrix $M$ for the right-handed neutrinos with three large eigenvalues, with $M_{1}<M_{2}<M_{3}$. If the largest eigenvalue of $h^{(n)}$ is $\mathcal{O}(1)$, one has $M_{3} \simeq v_{B-L}^{2} / M_{\mathrm{P}}$. The heavy Majorana neutrinos can be integrated out yielding for the matter part of the superpotential

$$
W_{M}=h_{i j}^{(u)} \mathbf{1 0}_{i} \mathbf{1 0}_{j} H_{u}+h_{i j}^{(d)} \overline{\mathbf{5}}_{i} \mathbf{1 0}_{j} H_{d}-\frac{1}{2}\left(h^{(\nu)} \frac{1}{M} h^{(\nu) T}\right)_{i j}\left(\overline{\mathbf{5}}_{i} H_{u}\right)\left(\overline{\mathbf{5}}_{j} H_{u}\right),
$$

with the familiar dimension-5 seesaw operator for light neutrino masses.

\footnotetext{
${ }^{3}$ For simplicity, we use a spectator chiral superfield to describe supersymmetry breaking. The field $Z$ is not essential for the connection between R-parity breaking and B-L breaking discussed in this section.
} 


\begin{tabular}{c|cccccccccc}
\hline \hline & $\mathbf{1 0}_{i}$ & $\mathbf{5}_{i}^{*}$ & $\mathbf{1}_{i}$ & $H_{u}$ & $H_{d}$ & $N$ & $N^{c}$ & $\Phi$ & $X$ & $Z$ \\
\hline$R$ & 1 & 1 & 1 & 0 & 0 & 0 & -2 & -1 & 4 & 0 \\
\hline \hline
\end{tabular}

Table 1: R-charges of matter fields, Higgs fields and $S O(10)$ singlets.

Since the field $\Phi$ carries R-charge -1, the VEV $\langle\Phi\rangle$ breaks R-parity, which is conserved by the VEV $\langle Z\rangle$. Thus, the breaking of B-L is tied to the breaking of R-parity. This is the key feature of the mechanism for R-parity breaking presented in this papent. The breaking of R-parity is transmitted to the low-energy degrees of freedom via higher-dimensional operators in the superpotential and the Kähler potential. The leading correction to the Kähler potential is

$$
\delta K_{1}=\frac{1}{M_{\mathrm{P}}{ }^{3}}\left(a_{i} Z^{\dagger}+a_{i}^{\prime} Z\right) \Phi^{\dagger} N^{c} \overline{\mathbf{5}}_{i} H_{u}+\frac{1}{M_{\mathrm{P}}{ }^{3}}\left(c_{i} Z^{\dagger}+c_{i}^{\prime} Z\right) \Phi N^{\dagger} \overline{\mathbf{5}}_{i} H_{u}+\text { h.c. } .
$$

Replacing the spectator fields $Z$ and $\Phi$, as well as $N^{c}$ by their expectation values, one obtains the correction to the superpotential

$$
\delta W_{1}=\mu_{i} \Theta \overline{\overline{5}}_{i} H_{u},
$$

with

$$
\mu_{i}=\mathcal{O}\left(m_{3 / 2}\right), \quad \Theta=\frac{v_{B-L}^{2}}{M_{\mathrm{P}}{ }^{2}} \simeq \frac{M_{3}}{M_{\mathrm{P}}},
$$

where $m_{3 / 2}=F_{Z} /\left(\sqrt{3} M_{\mathrm{P}}\right)$ is the gravitino mass. Note that $\Theta$ can be increased or decreased by an appropriate choice of Yukawa couplings in Eqs. (66) and (77). Eq. (12) is the familiar bilinear R-parity breaking term [3]. The correction to the Kähler potential

$$
\delta K_{0}=\frac{k}{M_{\mathrm{P}}} Z^{\dagger} H_{d} H_{u}+\text { h.c. }
$$

yields the corresponding R-parity conserving term [21]

$$
\delta W_{0}=\mu H_{d} H_{u}, \quad \mu=\mathcal{O}\left(m_{3 / 2}\right) .
$$

Note that $\mu$ and $\mu_{i}$ are generated by operators of different mass dimension. Hence, their values may easily differ by one or two orders of magnitude, allowing for $\mu>\mu_{i}, m_{3 / 2}$ and a gravitino LSP.

To analyse the complete superpotential including the R-symmetry breaking terms, it is convenient to perform a rotation of the Higgs and lepton superfields,

$$
H_{d}=H_{d}^{\prime}-\epsilon_{i} l_{i}^{\prime}, \quad l_{i}=l_{i}^{\prime}+\epsilon_{i} H_{d}^{\prime},
$$

\footnotetext{
${ }^{4}$ For a recent discussion of the connection between B-L breaking and R-parity breaking in the context of string compactifications, see [19]20].
} 
where $\epsilon_{i}=\mu_{i} \Theta / \mu$. In terms of the new fields the superpotential reads

$$
\begin{aligned}
W= & W_{M}+\delta W_{0}+\delta W_{1} \\
= & \mu H_{d}^{\prime} H_{u}+h_{i j}^{(u)} q_{i} u_{j}^{c} H_{u}+h_{i j}^{(d)} d_{i}^{c} q_{j} H_{d}^{\prime}+h_{i j}^{(e)} l_{i}^{\prime} e_{j}^{c} H_{d}^{\prime} \\
& -\epsilon_{k} h_{i j}^{(d)} d_{i}^{c} q_{j} l_{k}^{\prime}-\epsilon_{k} h_{i j}^{(e)} l_{i}^{\prime} e_{j}^{c} l_{k}^{\prime}-\frac{1}{2}\left(h^{(\nu)} \frac{1}{M} h^{(\nu) T}\right)_{i j}\left(l_{i}^{\prime} H_{u}\right)\left(l_{j}^{\prime} H_{u}\right)+\mathcal{O}\left(\epsilon^{2}, \epsilon m_{\nu}\right) .
\end{aligned}
$$

The mixing of Higgs and lepton superfields has induced trilinear R-parity breaking terms $\mathcal{O}(\epsilon)$. As we will discuss in Sec. 3, the mixing terms induce vacuum expectation values for the scalar neutrinos that in turn induce mixing terms $\mathcal{O}(\epsilon)$ of neutrinos with the neutralinos, and neutrino masses suppressed by $\mathcal{O}\left(\epsilon^{2}\right)$.

It is remarkable that the potentially dangerous operator leading to proton decay is strongly suppressed compared to the trilinear terms $\mathcal{O}(\epsilon)$ in Eq. (17). The leading operator is

$$
\delta W_{2}=\frac{1}{M_{\mathrm{P}}{ }^{5}} u^{c} d^{c} d^{c} N^{c} \Phi^{3} X .
$$

For global supersymmetry one has $\langle X\rangle=0$, which in supergravity is modified to $\langle X\rangle=$ $\mathcal{O}\left(m_{3 / 2}\right) 5$. One then obtains

$$
\delta W_{2} \propto \frac{m_{3 / 2} v_{B-L}^{4}}{M_{\mathrm{P}}^{5}} u^{c} d^{c} d^{c}+\ldots
$$

For $\lambda, \lambda^{\prime}$ satisfying Eq. (2), the coefficient of the dangerous dimension- $4 \Delta B=1$ operator is much smaller than the upper bound from the proton lifetime [4].

\subsection{Scale of B-L breaking and Thermal Leptogenesis}

The phenomenological viability of the model depends on the size of R-parity breaking mixings $\epsilon_{i}$ and therefore on the scale $v_{B-L}$ of R-parity breaking. An important constraint comes from baryogenesis. As already discussed in the introduction, the potential washout of a baryon asymmetry before the electroweak phase transition is avoided if the R-parity violating Yukawa couplings satisfy $\lambda_{i j k}, \lambda_{i j k}^{\prime} \lesssim 10^{-7}$, which in turn implies:

$$
\left(\frac{\epsilon_{i}}{10^{-6}}\right)\left(\frac{\tan \beta}{10}\right) \lesssim 1 .
$$

This is a sufficient condition, which can be relaxed for some flavour structures [5].

As an illustration for possible scales of B-L breaking we use a model [22] for quark and lepton mass hierarchies based on a Froggatt-Nielsen $U(1)$ flavour symmetry. The

\footnotetext{
${ }^{5}$ The VEV $\langle X\rangle=\mathcal{O}\left(m_{3 / 2}\right)$ also causes an additional contribution to the bilinear term via $\delta W=$ $\left(1 / M_{\mathrm{P}}{ }^{3}\right) X \Phi N^{c} \overline{\boldsymbol{5}}_{i} H_{u}$, which is comparable to those from $\delta K_{1}$.
} 


\begin{tabular}{c|cccccccccccccc}
\hline \hline$\psi_{i}$ & $\mathbf{1 0}_{3}$ & $\mathbf{1 0}_{2}$ & $\mathbf{1 0}_{1}$ & $\mathbf{5}_{3}^{*}$ & $\mathbf{5}_{2}^{*}$ & $\mathbf{5}_{1}^{*}$ & $\mathbf{1}_{3}$ & $\mathbf{1}_{2}$ & $\mathbf{1}_{1}$ & $H_{u}$ & $H_{d}$ & $\Phi$ & $X$ & $Z$ \\
\hline$Q_{i}$ & 0 & 1 & 2 & $\mathrm{a}$ & $\mathrm{a}$ & $\mathrm{a}+1$ & $\mathrm{~b}$ & $\mathrm{c}$ & $\mathrm{d}$ & 0 & 0 & 0 & 0 & 0 \\
\hline \hline
\end{tabular}

Table 2: Chiral charges: $a=0$ or 1 , and $0 \leq b \leq c \leq d$.

mass hierarchy is generated by the expectation value of a singlet field $\phi$ with charge $Q_{\phi}=-1$ via nonrenormalizable interactions with a scale $\Lambda=\langle\phi\rangle / \eta>\Lambda_{G U T}, \eta \simeq 0.06$. Yukawa couplings and bilinear terms for $S U(5)$ multiplets $\psi_{i}$ with charge $Q_{i}$ scale like

$$
h_{i j} \propto \eta^{Q_{i}+Q_{j}}, \quad \mu_{i} \propto \eta^{Q_{i}} .
$$

Charges $Q_{i}$ describing qualitatively the observed quark and lepton masses and mixings are listed in Table 2 . The model also predicts the observed baryon asymmetry via leptogenesis for the cases where $a+d=2$. There are two, at low energies indistinguishable, consistent scales of B-L breaking: $M_{3} \sim 10^{15} \mathrm{GeV}(a=b=0, c=1, d=2)$ and $M_{3} \sim 10^{12} \mathrm{GeV}(b=c=0, a=d=1)$. For $\mu_{i} / \mu=1.0 \ldots 0.01$ these two cases lead to the R-parity breaking mixing parameters (cf. Eq. (13))

$$
\text { (I) } \frac{\epsilon_{i}}{\eta^{Q_{i}}}=10^{-3} \ldots 10^{-5}, \quad \text { (II) } \frac{\epsilon_{i}}{\eta^{Q_{i}}}=10^{-6} \ldots 10^{-8} .
$$

In the extreme case $M_{3} \sim M_{2} \sim M_{1} \sim 10^{10} \mathrm{GeV}$ without Froggatt-Nielsen symmetry, where leptogenesis may still work for an appropriate enhancement of the CP asymmetry, one has

$$
\text { (III) } \epsilon_{i}=10^{-8} \ldots 10^{-10} .
$$

In the flavour models (I) and (II) the RPV mixings $\epsilon_{i}$ are suppressed by $\eta^{Q_{i}}$. As we shall see in the following section, model (I) is inconsistent with the constraints from neutrino masses and baryogenesis washout; the models (II) and (III) are consistent with both constraints.

The expected mass scale of right-handed neutrinos depends on the mechanism which breaks B-L. The expectation value of a field with lepton number $L=2$ can generate heavy Majorana masses via renormalizable Yukawa couplings. With B-L broken at the GUT scale, and for Yukawa coupling $\mathcal{O}(1)$ for the third family, one then obtains the canonical result $M_{3} \sim v_{B-L} \sim 10^{15} \mathrm{GeV}$. On the other hand, if right-handed neutrino masses are generated via a nonrenormalizable dimension-5 operator and the expectation value of a field with $L=1$, as in Eq. (6) , one has instead $M_{3} \sim v_{B-L}^{2} / M_{\mathrm{P}} \sim 10^{12} \mathrm{GeV}$. This illustrates how the two mass scales for $M_{3}$, which correspond to the two cases (I) and (II), respectively, might be obtained. 


\section{Neutrino Masses}

The model we are considering generates after supersymmetry breaking bilinear R-parity violating terms, Eq. (12), and tiny R-parity violating Yukawa couplings, Eq. (19), that we neglect in what follows. Scenarios with just bilinear R-parity violation have been thoroughly studied in the literature [23]. Here, we will limit ourselves to estimate the size of neutrino masses, following closely [24].

At the high-energy scale, the soft SUSY breaking Lagrangian reads

$$
-\mathcal{L}_{\text {soft }}=m_{H_{d}}^{2}\left|H_{d}\right|^{2}+m_{H_{u}}^{2}\left|H_{u}\right|^{2}+m_{l_{i}}^{2}\left|\tilde{l}_{i}\right|^{2}+\left(B H_{d} H_{u}+B_{i} \tilde{l}_{i} H_{u}+m_{l_{i} H_{d}}^{2} \tilde{l}_{i} H_{d}^{*}+\text { h.c. }\right)+\ldots
$$

For the computation of neutrino masses we find convenient to work in the basis where the R-parity violating bilinear couplings in the superpotential are rotated away, $\mu_{i}=0$, through the field redefinition Eq. (16). This choice of basis has the advantage that once the basis has been fixed at the high energy scale, the condition $\mu_{i}=0$ holds at any scale, and it is not necessary to redefine the basis again at low energies. We also choose the phases of the lepton doublets such that the $\epsilon_{i}$ are real. In this basis the soft SUSY breaking Lagrangian is given by

$$
-\mathcal{L}_{\text {soft }}=m_{H_{d}^{\prime}}^{2}\left|H_{d}^{\prime}\right|^{2}+m_{H_{u}}^{2}\left|H_{u}\right|^{2}+\left.m_{l_{i}^{\prime}}^{2} \tilde{l}_{i}^{\prime}\right|^{2}+\left(B^{\prime} H_{d}^{\prime} H_{u}+B_{i}^{\prime} \tilde{l}_{i}^{\prime} H_{u}+m_{l_{i}^{\prime} H_{d}^{\prime}}^{2} \tilde{l}_{i}^{\prime} H_{d}^{\prime *}+\text { h.c. }\right)+\ldots
$$

where

$$
\begin{gathered}
m_{H_{d}^{\prime}}^{2}=m_{H_{d}}^{2}+\epsilon_{i} \operatorname{Re}\left(m_{l_{i} H_{d}}^{2}\right)+\mathcal{O}\left(\left|\epsilon_{i}\right|^{2}\right), \\
m_{l_{i}^{\prime}}^{2}=m_{l_{i}}^{2}-\epsilon_{i} \operatorname{Re}\left(m_{l_{i} H_{d}}^{2}\right)+\mathcal{O}\left(\left|\epsilon_{i}\right|^{2}\right), \\
B^{\prime}=B+B_{i} \epsilon_{i}, \\
B_{i}^{\prime}=B_{i}-B \epsilon_{i}, \\
m_{l_{i}^{\prime} H_{d}^{\prime}}^{2}=m_{l_{i} H_{d}}^{2}+\epsilon_{i}\left(m_{l_{i}}^{2}-m_{H_{d}}^{2}\right)+\mathcal{O}\left(\left|\epsilon_{i}\right|^{2}\right) .
\end{gathered}
$$

Minimisation of the scalar potential yields non-vanishing vacuum expectation values for the neutral components of the Higgs doublets, as well as for the sneutrinos,

$$
\left\langle\tilde{\nu}_{i}^{\prime}\right\rangle=\frac{B_{i}^{\prime} \tan \beta+m_{l_{i}^{\prime} H_{d}^{\prime}}^{2}}{m_{l_{i}^{\prime}}^{2}-\frac{1}{2} M_{Z}^{2} \cos 2 \beta}\left\langle H_{d}^{\prime}\right\rangle, \quad v_{\nu}^{2}=\sum_{i=1}^{3}\left\langle\tilde{\nu}_{i}^{\prime}\right\rangle^{2} .
$$

These vacuum expectation values induce mixings between neutrinos and gauginos, giving rise to one non-vanishing neutrino mass through an "electroweak" seesaw mechanism. The resulting neutrino mass is

$$
m_{\nu}^{R_{p}}=\frac{1}{2} g_{Z}^{2} v_{\nu}^{2} \sum_{\alpha=1}^{4} \frac{\left|c_{\tilde{z} \alpha}\right|^{2}}{m_{\chi_{\alpha}^{0}}}
$$


where $g_{Z}$ is the Z-boson gauge coupling, $m_{\chi_{\alpha}^{0}}$ are the neutralino masses and $c_{\tilde{z} \alpha}$ is the Zino component of the neutralino $\chi_{\alpha}^{0}$. In the following estimates we will replace the sum over inverse neutralino masses by the inverse of the characteristic SUSY breaking scale, $1 / \widetilde{m}$.

The size of the neutrino masses depends crucially on the mechanism for supersymmetry breaking. Generically, one expects

$$
B_{i}^{\prime} \sim \mu_{i} \widetilde{m} \sim \epsilon_{i} \widetilde{m}^{2}, \quad m_{l_{i}^{\prime} H_{d}^{\prime}}^{2}=\epsilon_{i} \widetilde{m}^{2} .
$$

Then, using Eqs. (27) and (28) we find for $\epsilon_{1}, \epsilon_{2} \leq \epsilon_{3}$,

$$
m_{\nu}^{R_{p}} \sim 10^{-4} \mathrm{eV}\left(\frac{\epsilon_{3}}{10^{-7}}\right)^{2}\left(\frac{\widetilde{m}}{200 \mathrm{GeV}}\right)^{-1} .
$$

We can now insert the values of $\epsilon_{3}$ for the different flavour models considered in the previous section. Clearly, model (I), which has a high scale of B-L breaking, is excluded. In models (II) and (III) one has $\epsilon_{3}<10^{-7}$ and $\epsilon_{3}<10^{-8}$, respectively. Here the neutrino mass terms induced by R-parity breaking are negligible and the baryogenesis constraint, Eq. (20), is fulfilled for all values of $\tan \beta$.

\section{Cosmology and Collider Physics}

\subsection{Gravitino Decay}

Since R-parity is broken, the gravitino is no more stable in our setting, but it still has a lifetime much longer than the age of the universe, since it is suppressed both by the Planck mass and the small R-parity breaking parameters.

The two-body decay is determined by the mixing of the neutralinos with the neutrinos. Neglecting the small neutrino masses, one has [6]

$$
\Gamma\left(\psi_{3 / 2} \rightarrow \gamma \nu\right)=\frac{1}{32 \pi}\left|U_{\tilde{\gamma} \nu}\right|^{2} \frac{m_{3 / 2}^{3}}{M_{\mathrm{P}}^{2}} .
$$

The photino-neutrino mixing can be approximated by (cf. Eq. (27))

$$
\left|U_{\tilde{\gamma} \nu}\right| \simeq g_{z}\left|\sum_{\alpha=1}^{4} c_{\tilde{\gamma} \alpha} c_{\tilde{z} \alpha}^{*} \frac{v_{\nu}}{m_{\chi_{\alpha}^{0}}}\right| \sim 10^{-8}\left(\frac{\epsilon_{3}}{10^{-7}}\right)\left(\frac{\widetilde{m}}{200 \mathrm{GeV}}\right)^{-1}
$$

for $\epsilon_{1}, \epsilon_{2} \leq \epsilon_{3}$, where we made the rough estimate $0.1 / \widetilde{m}$ for the weighted sum of neutralino masses and the coupling, taking into account that not all mixings can be maximal. Using $M_{\mathrm{P}}=2.4 \times 10^{18} \mathrm{GeV}$, one obtains for the gravitino lifetime [6]

$$
\tau_{3 / 2}^{2-\text { body }} \simeq 4 \times 10^{27} \mathrm{~s}\left(\frac{\epsilon_{3}}{10^{-7}}\right)^{-2}\left(\frac{\widetilde{m}}{200 \mathrm{GeV}}\right)^{2}\left(\frac{m_{3 / 2}}{10 \mathrm{GeV}}\right)^{-3}
$$


The three-body decay is usually subdominant due to the phase-space and intermediate heavy particle suppression. For the decay with intermediate heavy $\tilde{\tau}_{R}$, neglecting all external masses in the phase space factor, we find

$$
\Gamma\left(\psi_{3 / 2} \rightarrow \tau_{R} l_{i} l_{j}\right)=\frac{\left|\lambda_{i j 3}\right|^{2}}{3(32)^{2} \pi^{3}} \frac{m_{3 / 2}^{3}}{M_{P}^{2}} F\left(\frac{m_{\tilde{\tau}_{R}}}{m_{3 / 2}}\right),
$$

where

$$
F(\alpha)=\int_{0}^{1} d x \frac{x^{3}(1-x)^{2}}{\left(1-x-\alpha^{2}\right)^{2}} \simeq \frac{1}{60 \alpha^{4}} .
$$

The full expression has been obtained in [25].

In the case where only bilinear R-parity breaking is present, the $\lambda_{i j k}$ couplings are generated from the Yukawa couplings as

$$
\lambda_{i j k}=\epsilon_{i} h_{j k}^{(e)} .
$$

Then the inverse partial width for the three-body decay,

$$
\Gamma\left(\psi_{3 / 2} \rightarrow \tau_{R} l_{i} l_{j}\right)^{-1} \simeq 4 \times 10^{37} s\left(\frac{\epsilon_{3}}{10^{-7}}\right)^{-2}\left(\frac{\tan \beta}{10}\right)^{-2}\left(\frac{\widetilde{m}}{200 \mathrm{GeV}}\right)^{4}\left(\frac{m_{3 / 2}}{10 \mathrm{GeV}}\right)^{-7},
$$

is much larger than the lifetime determined from the two-body decay, Eq. (33), as long as the mixing between photino and neutrino is not unnaturally suppressed.

\subsection{Extragalactic Diffuse Gamma-Ray Emission}

A stringent astrophysical constraint for decaying gravitino dark matter is the measured gamma-ray flux [6]. Assuming that the gravitino constitutes the dominant component of dark matter, its decay into neutrino and photon gives rise to an extragalactic diffuse gamma-ray flux with a characteristic energy spectrum, corresponding to a red shifted monochromatic line. A photon with measured energy $E=m_{3 / 2} /(2(1+z))$ has been emitted at the comoving distance $\chi(z)$, with $d \chi / d z=(1+z)^{-3 / 2} /\left(a_{0} H_{0} \sqrt{\Omega_{M}\left(1+\kappa(1+z)^{-3}\right)}\right)$. Here $a_{0}$ and $H_{0}$ are the present scale factor and Hubble parameter, respectively, and $\kappa=\Omega_{\Lambda} / \Omega_{M} \simeq 3$, with $\Omega_{\Lambda}+\Omega_{M}=1$, assuming a flat universe. For the photon flux one obtains, for $\tau_{3 / 2} \gg H_{0}^{-1}$,

$$
E^{2} \frac{d J_{e g}}{d E}=C_{\gamma}\left(1+\kappa\left(\frac{2 E}{m_{3 / 2}}\right)^{3}\right)^{-1 / 2}\left(\frac{2 E}{m_{3 / 2}}\right)^{5 / 2} \theta\left(1-\frac{2 E}{m_{3 / 2}}\right),
$$

with

$$
C_{\gamma}=\frac{\Omega_{3 / 2} \rho_{c}}{8 \pi \tau_{3 / 2} H_{0} \Omega_{M}^{1 / 2}}=10^{-6}\left(\mathrm{~cm}^{2} \mathrm{str} \mathrm{s}\right)^{-1} \mathrm{GeV}\left(\frac{\tau_{3 / 2}}{10^{28 \mathrm{~S}}}\right)^{-1}
$$


here $\tau_{3 / 2}$ is given by Eq. (33), and we have taken the gravitino density equal to the Cold Dark Matter density as $\Omega_{3 / 2} h^{2}=0.1, \rho_{c}=1.05 h^{2} \times 10^{-5} \mathrm{GeVcm}^{-3}, \Omega_{M}=0.25$ and $H_{0}=h 100 \mathrm{~km} \mathrm{~s}^{-1} \mathrm{Mpc}^{-1}$ with $h=0.73$ [26].

In addition to the extragalactic signal one also expects a sharp line from the halo of our galaxy with an intensity comparable to the extragalactic signal and strong anisotropy [27]. We have in fact from the decay of halo gravitinos

$$
E^{2} \frac{d J_{\text {halo }}}{d E}=D_{\gamma} \delta\left(1-\frac{2 E}{m_{3 / 2}}\right)
$$

where

$$
D_{\gamma}=C_{\gamma} \frac{H_{0} \Omega_{M}^{1 / 2}}{\Omega_{3 / 2} \rho_{c}} \int_{\text {l.o.s. }} \rho_{\text {halo }}(\vec{l}) d \vec{l} .
$$

The ratio $D_{\gamma} / C_{\gamma}$ is given only by cosmological constants and the halo dark matter density integrated along the line of sight, so the intensity and angular distribution of the halo signal is very sensitive to the distribution of the dark matter in the Milky Way. It is surprising that for typical halo models, such number is of order unity [27] and shows moderate angular dependence if one excludes the galactic centre region. The anisotropic part of the halo signal may be partially hidden in the diffuse galactic $\gamma$-ray emission due to conventional astrophysical processes. We expect therefore the isotropic signal in the extragalactic $\gamma$-ray flux to be a combination of both the continuum spectrum in Eq. (38) and part of the halo line in Eq. (40).

Assuming that one understands the diffuse galactic $\gamma$-ray flux, one can extract from the EGRET data the extragalactic diffuse component. The first analysis of Sreekumar et al. 28] gave an extragalactic flux described by the power law

$$
E^{2} \frac{d J}{d E}=1.37 \times 10^{-6}\left(\frac{E}{1 \mathrm{GeV}}\right)^{-0.1}\left(\mathrm{~cm}^{2} \operatorname{str} \mathrm{s}\right)^{-1} \mathrm{GeV}
$$

in the energy range $50 \mathrm{MeV}-10 \mathrm{GeV}$. A non-observation of a $\gamma$-ray line can then be used to constrain the allowed gravitino mass and lifetime [6]. Assuming the gravitinos to make up all the Cold Dark Matter density, and taking a $3 \sigma$ upper bound on the flux above 100 $\mathrm{MeV}$ corresponding to $2.23 \times 10^{-6}\left(\mathrm{~cm}^{2} \operatorname{str~s}\right)^{-1} \mathrm{GeV}$ [28], we can have directly a rough bound on the gravitino lifetime from $C_{\gamma}$ as

$$
\tau_{3 / 2} \gtrsim 4 \times 10^{27} \mathrm{~s}
$$

The more recent analysis of the EGRET data [29] shows in the $50 \mathrm{MeV}-2 \mathrm{GeV}$ range a power law behaviour, but a clear excess between $2 \mathrm{GeV}$ and $10 \mathrm{GeV}$. The maximal flux allowed by the data taking into account the model dependence and systematic errors is not very far from the one obtained in the old analysis, in fact the integrated 
flux between $0.1-10 \mathrm{GeV}$ is given as $(11.1 \pm 0.1) \times 10^{-6} \mathrm{~cm}^{-2} \mathrm{str}^{-1} \mathrm{~s}^{-1}$ compared to $(14.5 \pm 0.5) \times 10^{-6} \mathrm{~cm}^{-2} \mathrm{str}^{-1} \mathrm{~s}^{-1}[29]$.

This is precisely the energy range where, based on our lower bound on the gravitino mass of $5 \mathrm{GeV}$, one may expect a gravitino signal. It is very remarkable that also the measured flux corresponds to the expectation of the model for R-parity and B-L breaking discussed in Sec. 2 as can be seen from the bound Eq. (43). On the other hand we would expect also an anisotropic flux from the halo component that EGRET does not resolve probably due to the galactic background, which is an order of magnitude larger than the extracted extragalactic signal.

The excess in the extragalactic $\gamma$-ray flux above $2 \mathrm{GeV}$ from the EGRET data [29] has also been related to the annihilation of heavy neutralinos in the galactic halo [30]. Due to the current limitations in the determination of the diffuse galactic $\gamma$-ray emission [31] theoretical interpretations of the EGRET excess remain uncertain at present. Clarification can be expected from the Gamma Ray Large Area Space Telescope (GLAST) [32], to be launched this fall, that aims to improve by a factor 30-50 the sensitivity of the EGRET satellite to the diffuse gamma ray flux in the range $20 \mathrm{MeV}-10 \mathrm{GeV}$.

Another constraint comes from the neutrino flux. In the energy range of interest, from about $1 \mathrm{GeV}$ to $1 \mathrm{TeV}$, the extraterrestrial neutrino flux is constrained by the flux of upward-going muons measured by the IMB experiment, that does not show any discrepancy with respect to the expected neutrino flux from cosmic ray interactions in the atmosphere. The requirement that the neutrino flux from gravitino decay does not exceed the observed flux, translates into a lower bound on the gravitino lifetime, which is roughly $\tau_{3 / 2} \gtrsim 6 \times 10^{24} \mathrm{~s}$ for $m_{3 / 2}=1 \mathrm{TeV}$, and becomes weaker for smaller gravitino masses [33]. This bound is clearly consistent with a signal in the EGRET data, as discussed above.

\subsection{Collider Signatures}

The collider signatures depend on the nature of the NLSP. Here we consider the cases that the NLSP is the lightest stau or the lightest neutralino.

The lightest stau, that we assume mainly right-handed, decays through $\widetilde{\tau}_{R} \rightarrow$ $\tau \nu_{\mu}, \mu \nu_{\tau}$. On the other hand, the small left-handed component of the stau mass eigenstate can trigger a decay into two jets through $\widetilde{\tau}_{L} \rightarrow b^{c} t$, provided the process is kinematically open. The hadronic decays are enhanced compared to the leptonic decays by

the larger bottom Yukawa coupling and by the colour factor, but are usually suppressed by the small left-right mixing.

If the leptonic decay channel is the dominant mode, the decay length can be approx- 
imated by

$$
c \tau_{\tilde{\tau}}^{l e p} \sim 30 \mathrm{~cm}\left(\frac{m_{\tilde{\tau}}}{200 \mathrm{GeV}}\right)^{-1}\left(\frac{\epsilon_{2}}{10^{-7}}\right)^{-2}\left(\frac{\tan \beta}{10}\right)^{-2} .
$$

It is intriguing that the sufficient condition to avoid the erasure of the baryon asymmetry, Eq. (20), implies the observation of a displaced stau vertex at future colliders, more than $3 \mathrm{~mm}$ away from the beam axis for $\epsilon_{2}<10^{-6}$. In the particular case of the flavour model (II) discussed in Sec. 2.2, $\epsilon_{2} \sim 6 \times 10^{-8}$, one has a spectacular signal consisting on a heavily ionising charged track of length $\sim 0.8 \mathrm{~m}$, followed by a muon track or a jet and missing energy, corresponding to $\widetilde{\tau} \rightarrow \mu \nu_{\tau}$ or $\widetilde{\tau} \rightarrow \tau \nu_{\mu}$, respectively.

If the hadronic channel $\widetilde{\tau}_{L} \rightarrow b^{c} t$ is the dominant mode, the decay length is given by

$$
c \tau_{\tilde{\tau}}^{h a d} \sim 1.4 \mathrm{~m}\left(\frac{m_{\tilde{\tau}}}{200 \mathrm{GeV}}\right)^{-1}\left(\frac{\epsilon_{3}}{10^{-7}}\right)^{-2}\left(\frac{\tan \beta}{10}\right)^{-2}\left(\frac{\cos \theta_{\tau}}{0.1}\right)^{-2}
$$

where $\theta_{\tau}$ denotes the mixing angle of the staus. This channel also yields a very unique signature at colliders, consisting of a heavily ionising charged track followed by two jets.

These characteristic signatures would allow to distinguish at colliders our scenario from the case with conserved R-parity where the decay $\tilde{\tau} \rightarrow \psi_{3 / 2} \tau$ leads to (cf. [34])

$$
c \tau_{\tilde{\tau}}^{3 / 2} \sim 40 \mathrm{~cm}\left(\frac{m_{3 / 2}}{1 \mathrm{keV}}\right)^{2}\left(\frac{m_{\tilde{\tau}}}{200 \mathrm{GeV}}\right)^{-5}
$$

Hence, for a gravitino mass $m_{3 / 2} \lesssim \mathcal{O}(10 \mathrm{keV})$, the decay length of the lightest stau is shorter than $\mathcal{O}(10 \mathrm{~m})$, and would therefore decay inside the detector into tau and gravitino. The experimental signature for this process would be identical to the decay $\widetilde{\tau} \rightarrow \tau \nu_{\mu}$. However, the scenario with R-parity violation also predicts the decay $\widetilde{\tau} \rightarrow \mu \nu_{\tau}$, with very similar branching ratio due to $S U(2)$ invariance. Although this signature could be mimicked by a scenario with conserved R-parity if lepton flavour is violated, through the decay $\tilde{\tau} \rightarrow \mu \psi_{3 / 2}$, large branching ratios are precluded from present bounds on flavour violation [35]. In consequence, the observation of a comparable number of tau and muon events in stau decays would constitute a signature for the scenario with Rparity violation. Also, the observation of a stau decaying into two jets would undoubtedly point to the scenario with R-parity violation.

On the other hand, if the lightest neutralino is the NLSP, it decays through $\chi_{1}^{0} \rightarrow$ $\tau^{ \pm} W^{\mp}$ [36], or through $\chi_{1}^{0} \rightarrow b b^{c} \nu$ [37] if the former decay channel is kinematically closed. The corresponding decay lengths can be approximated by

$$
\begin{aligned}
c \tau_{\chi_{1}^{0}}^{2-\text { body }} & \sim 20 \mathrm{~cm}\left(\frac{m_{\chi_{1}^{0}}}{200 \mathrm{GeV}}\right)^{-3}\left(\frac{\epsilon_{3}}{10^{-7}}\right)^{-2}\left(\frac{\tan \beta}{10}\right)^{2}, \\
c \tau_{\chi_{1}^{0}}^{3-\text { body }} & \sim 600 \mathrm{~m}\left(\frac{m_{\widetilde{\nu}_{L}}}{300 \mathrm{GeV}}\right)^{4}\left(\frac{m_{\chi_{1}^{0}}}{200 \mathrm{GeV}}\right)^{-5}\left(\frac{\epsilon_{3}}{10^{-7}}\right)^{-2}\left(\frac{\tan \beta}{10}\right)^{-2} .
\end{aligned}
$$


Again, this scenario can be easily discriminated at future colliders from the scenario with conserved R-parity. In this case, the neutralino decays into gravitino and photon [38] with decay length

$$
c \tau_{\chi_{1}^{0}}^{3 / 2} \sim 80 \mathrm{~cm}\left(\frac{m_{3 / 2}}{1 \mathrm{keV}}\right)^{2}\left(\frac{m_{\chi_{1}^{0}}}{200 \mathrm{GeV}}\right)^{-5}
$$

For a gravitino mass $m_{3 / 2} \lesssim \mathcal{O}(10 \mathrm{keV})$ the neutralino would decay inside the detector producing an energetic photon and missing energy, which is clearly distinguishable from the signals in the R-parity violating scenario that in general involve jets.

\subsection{Microscopic Determination of the Planck Mass}

Recently, a method has been proposed for the microscopic determination of the Planck mass in collider experiments [34, providing a direct test of supergravity. The method requires a very long lived stau NLSP which decays mostly into tau and gravitino, which is difficult to reconcile with recent constraints from BBN [79], unless there is a late-time entropy production [10]. In the picture proposed in this letter, where primordial nucleosynthesis, thermal leptogenesis and dark matter are naturally consistent, this method cannot be pursued, as the stau decays predominantly in the R-parity violating channel into charged lepton and neutrino.

Nevertheless, from a gravitino signal in the diffuse $\gamma$-ray flux and the width for the stau decay into two jets, one can still obtain a microscopic estimate of the Planck mass. The gravitino mass is given by the maximal energy of the photon, $m_{3 / 2}=2 E_{\gamma}$, and the gravitino lifetime can be determined from the photon flux, Eqs. (38,40). Then, using the expression for the gravitino decay rate, Eq. (31), one can rewrite the Planck mass in terms of the gravitino mass, lifetime and photino-neutrino mixing as

$$
\begin{aligned}
M_{\mathrm{P}} & =\left(\frac{m_{3 / 2}^{3} \tau_{3 / 2}}{32 \pi}\right)^{1 / 2}\left|U_{\tilde{\gamma} \nu}\right| \\
& =2.5 \times 10^{18} \mathrm{GeV}\left(\frac{m_{3 / 2}}{10 \mathrm{GeV}}\right)^{3 / 2}\left(\frac{\tau_{3 / 2}}{4 \times 10^{27} \mathrm{~s}}\right)^{1 / 2}\left(\frac{\left|U_{\tilde{\gamma} \nu}\right|}{10^{-8}}\right)
\end{aligned}
$$

where $\left|U_{\tilde{\gamma} \nu}\right|$ is related to the decay rate of the stau into two jets 6 . We can cast the dependence on the decay rate as a dependence on the decay length of the stau in this channel, yielding

$$
\left|U_{\tilde{\gamma} \nu}\right|^{2} \simeq 10^{-16}\left(\frac{c \tau_{\tilde{\tau}}^{\text {had }}}{1.4 \mathrm{~m}}\right)^{-1}\left(\frac{\widetilde{m}}{200 \mathrm{GeV}}\right)^{-3}\left(\frac{\tan \beta}{10}\right)^{2}\left(\frac{\cos \theta_{\tau}}{0.1}\right)^{2}
$$

\footnotetext{
${ }^{6}$ Note that the decay rate of the stau into leptons depends on $\epsilon_{2}$ whereas $\left|U_{\tilde{\gamma} \nu}\right|$ depends on the sneutrino VEV and therefore mainly on $\epsilon_{3}$ for the hierarchical case.
} 
The measurement of the decay length of the stau in the hadronic channel, complemented with additional information about supersymmetry breaking parameters, can provide a determination of $\left|U_{\tilde{\gamma} \nu}\right|$. The measurement of the photon energy and the photon flux in the diffuse $\gamma$-ray background then gives the gravitino mass and lifetime and, using Eq. (50), an estimate of the Planck mass.

\section{Conclusions}

On theoretical grounds, theories with and without R-parity are on equal footing. In this paper we have presented a simple model where R-parity is not conserved and its violation is connected to the scale of B-L breaking. One can then have R-parity violating couplings that are small enough to be consistent with baryogenesis and gravitino dark matter, yet large enough to allow for the NLSP decay before nucleosynthesis. For gravitino masses above $5 \mathrm{GeV}$ one obtains a cosmological history consistent with thermal leptogenesis, thermally produced gravitino dark matter and primordial nucleosynthesis.

Relic gravitino decays into neutrino and photon yield a diffuse halo and extragalactic $\gamma$-ray flux which depends on the R-parity violating Yukawa couplings. It is remarkable that for a gravitino mass $m_{3 / 2}=\mathcal{O}(10) \mathrm{GeV}$, the predicted photon flux could be part of the apparent excess in the extragalactic diffuse $\gamma$-ray flux obtained from the EGRET data. However, given the current uncertainties in the determination of the diffuse galactic $\gamma$-ray emission, this consistency may be accidental. Unequivocal evidence for decaying gravitino dark matter could come from the results of GLAST.

The flavour dependent pattern of R-parity breaking can give striking signatures at the LHC, in particular a vertex of the NLSP, that is significantly displaced from the beam axis. Together with the measurement of supersymmetry breaking parameters at the LHC, the observation of a redshifted photon spectral line from gravitino decay by GLAST can allow a microscopic determination of the Planck mass. In the less optimistic case where the R-parity breaking Yukawa couplings are near to their lower bound, astro-

physical detection will be very challenging whereas signals hinting at R-parity breaking and gravitino dark matter could still come from stau decays, as in the case of R-parity conservation.

\section{Acknowledgements}

We are grateful to G. Heinzelmann and J. Ripken for valuable discussions on the diffuse $\gamma$-ray background. L. C. acknowledges the support of the "Impuls- und Vernetzungsfond" 
of the Helmholtz Association, contract number VH-NG-006.

\section{References}

[1] P. Fayet, Supersymmetric Theories of Particles, Orbis Scientiae 1978:0413 (QCD161:C6:1978)

[2] N. Sakai and T. Yanagida, Nucl. Phys. B 197 (1982) 533;

S. Weinberg, Phys. Rev. D 26 (1982) 287.

[3] L. J. Hall and M. Suzuki, Nucl. Phys. B 231 (1984) 419.

[4] For recent discussions and references, see

B. C. Allanach, A. Dedes and H. K. Dreiner, Phys. Rev. D 69 (2004) 115002 [Erratum-ibid. D 72 (2005) 079902], [arXiv:hep-ph/0309196];

R. Barbier et al., Phys. Rept. 420 (2005) 1 [arXiv:hep-ph/0406039].

[5] B. A. Campbell, S. Davidson, J. R. Ellis, K. A. Olive, Phys. Lett. B 256 (1991) 457 ;

W. Fischler, G. F. Giudice, R. G. Leigh and S. Paban, Phys. Lett. B 258 (1991) 45 ;

H. K. Dreiner and G. G. Ross, Nucl. Phys. B $4 \mathbf{4 1 0}$ (1993) 188 [arXiv:hep-ph/9207221].

[6] F. Takayama and M. Yamaguchi, Phys. Lett. B 485 (2000) 388 [arXiv:hep-ph/0005214].

[7] M. Pospelov, arXiv:hep-ph/0605215;

R. H. Cyburt et al., JCAP 0611 (2006) 014 [arXiv:astro-ph/0608562].

[8] K. Kohri and F. Takayama, arXiv:hep-ph/0605243;

M. Kaplinghat and A. Rajaraman, Phys. Rev. D $\mathbf{7 4}$ (2006) 103004 [arXiv:astro-ph/0606209].

[9] M. Kawasaki, K. Kohri and T. Moroi, Phys. Rev. D 71 (2005) 083502 [arXiv:astro-ph/0408426];

J. L. Feng, S. Su and F. Takayama, Phys. Rev. D 70 (2004) 075019 [arXiv:hep-ph/0404231];

F. D. Steffen, JCAP 0609 (2006) 001 [arXiv:hep-ph/0605306].

[10] W. Buchmüller, K. Hamaguchi, M. Ibe and T. T. Yanagida, arXiv hep-ph/0605164. 
[11] T. Kanzaki, M. Kawasaki, K. Kohri and T. Moroi, Phys. Rev. D 75 (2007) 025011 [arXiv:hep-ph/0609246].

[12] M. Fukugita and T. Yanagida, Phys. Lett. B174, 45 (1986).

[13] S. Davidson and A. Ibarra, Phys. Lett. B 535 (2002) 25 [arXivihep-ph/0202239].

[14] W. Buchmüller, P. Di Bari and M. Plümacher, Annals Phys. 315 (2005) 305 [arXiv:hep-ph/0401240].

[15] M. Bolz, A. Brandenburg and W. Buchmüller, Nucl. Phys. B 606 (2001) 518 [arXiv:hep-ph/0012052].

[16] J. Pradler and F. D. Steffen, Phys. Rev. D 75 (2007) 023509 [arXiv:hep-ph/0608344].

[17] V. S. Rychkov and A. Strumia, arXiv:hep-ph/0701104.

[18] T. Asaka, W. Buchmüller and L. Covi, Phys. Lett. B 563 (2003) 209 [arXiv:hep-ph/0304142].

[19] R. Tatar and T. Watari, Nucl. Phys. B 747 (2006) 212 [arXiv:hep-th/0602238]; arXiv:hep-ph/0605315.

[20] W. Buchmüller, K. Hamaguchi, O. Lebedev and M. Ratz, arXiv:hep-th/0606187.

[21] G. F. Giudice and A. Masiero, Phys. Lett. B 206 (1988) 480.

[22] W. Buchmüller and T. Yanagida, Phys. Lett. B 445 (1999) 399 [arXiv:hep-ph/9810308].

[23] S. Roy and B. Mukhopadhyaya, Phys. Rev. D 55 (1997) 7020 [arXiv:hep-ph/9612447];

M. Hirsch, M. A. Diaz, W. Porod, J. C. Romao and J. W. F. Valle, Phys. Rev. D 62 (2000) 113008 [Erratum-ibid. D 65 (2002) 119901], [arXiv:hep-ph/0004115];

A. Abada, S. Davidson and M. Losada, Phys. Rev. D 65 (2002) 075010 [arXiv:hep-ph/0111332];

E. J. Chun, D. W. Jung and J. D. Park, Phys. Lett. B 557 (2003) 233 [arXiv:hep-ph/0211310].

[24] F. Takayama and M. Yamaguchi, Phys. Lett. B $476 \quad$ (2000) 116 [arXiv:hep-ph/9910320].

[25] G. Moreau and M. Chemtob, Phys. Rev. D 65 (2002) 024033 [arXiv:hep-ph/0107286]. 
[26] W.-M. Yao et al., Journal of Physics G 33, 1 (2006).

[27] T. Asaka, J. Hashiba, M. Kawasaki and T. Yanagida, Phys. Rev. D 58 (1998) 023507 [arXiv:hep-ph/9802271].

[28] P. Sreekumar et al. [EGRET Collaboration], Astrophys. J. 494 (1998) 523 [arXiv:astro-ph/9709257].

[29] A. W. Strong, I. V. Moskalenko and O. Reimer, Astrophys. J. 613 (2004) 962 [arXiv:astro-ph/0406254]; Astrophys. J. 613 (2004) 956. [arXiv astro-ph/0405441].

[30] D. Elsässer and K. Mannheim, Phys. Rev. Lett. 94 (2005) 171302 [arXiv:astro-ph/0405235].

[31] I. V. Moskalenko, S. W. Digel, T. A. Porter, O. Reimer and A. W. Strong, arXiv:astro-ph/0609768.

[32] http://www.glast.gsfc.nasa.gov.

[33] P. Gondolo, G. Gelmini and S. Sarkar, Nucl. Phys. B 392 (1993) 111 [arXiv hep-ph/9209236].

[34] W. Buchmüller, K. Hamaguchi, M. Ratz, T. Yanagida, Phys. Lett. B 588 (2004) 90 [arXiv:hep-ph/0402179].

[35] K. Hamaguchi and A. Ibarra, JHEP 0502 (2005) 028 [arXiv hep-ph/0412229].

[36] B. Mukhopadhyaya, S. Roy and F. Vissani, Phys. Lett. B 443 (1998) 191 [arXiv:hep-ph/9808265];

E. J. Chun and J. S. Lee, Phys. Rev. D 60, 075006 (1999) [arXiv;hep-ph/9811201].

[37] H. K. Dreiner and G. G. Ross, Nucl. Phys. B 365, 597 (1991).

[38] S. Dimopoulos, M. Dine, S. Raby and S. D. Thomas, Phys. Rev. Lett. 76 (1996) 3494 [arXiv:hep-ph/9601367]. 